\title{
Airway epithelial cell apoptosis and inflammation in COPD, smokers and
} nonsmokers

\author{
David M. Comer*, Joseph C. Kidney" ${ }^{\#}$, Madeleine Ennis ${ }^{\tau_{,}+}$and J. Stuart Elborn ${ }^{\uparrow,+}$
}

ABSTRACT: We hypothesised that primary bronchial epithelial cells (PBECs) from subjects with chronic obstructive pulmonary disease (COPD) respond differently to Pseudomonas aeruginosa lipopolysaccharide (LPS) after cigarette smoke extract (CSE) exposure than PBECs obtained from smokers without airflow obstruction and nonsmokers.

PBECs from 16 COPD subjects, 10 smokers without airflow obstruction and nine nonsmokers were cultured at air-liquid interface. Cultures were incubated with CSE prior to stimulation with P. aeruginosa LPS. Interleukin (IL)-6 and IL-8 were measured by ELISA and Toll-like receptor (TLR)-4 expression by fluorescence-activated cell sorter. Activation of nuclear factor (NF)- $\mathrm{B}$ was determined by Western blotting and ELISA, and MAPK and caspase-3 activity by Western blotting. Apoptosis was evaluated using Annexin-V staining and the terminal transferase-mediated dUTP nick end-labelling methods.

Constitutive release of IL-8 and IL-6 was greatest from the COPD cultures. However, CSE pretreatment followed by $P$. aeruginosa LPS stimulation reduced IL-8 release from COPD PBECs, but increased it from cells of smokers without airflow obstruction and nonsmokers. TLR-4 expression, MAPK and NF-кB activation in COPD cultures were reduced after CSE treatment, but not in the smokers without airflow obstruction or nonsmoker groups, which was associated with increased apoptosis.

CSE attenuates inflammatory responses to LPS in cells from people with COPD but not those from nonsmoking individuals and those who smoke without airflow obstruction.

KEYWORDS: Airway inflammation, cigarette smoking, chronic obstructive pulmonary disease, epithelial cell biology, infections in COPD, smoking and health

hronic obstructive pulmonary disease (COPD) is a global health problem and a major cause of death [1]. Exacerbations of COPD secondary to infection are a significant cause of morbidity, mortality and burden on healthcare costs. The relationship between smoking, infection, inflammation and COPD is not well understood.

Interleukin (IL)-6 and IL-8 responses of primary bronchial epithelial cells (PBECs) to stimulation with cigarette smoke extract (CSE) have been of particular interest [2]. IL-8 is an important neutrophil chemoattractant and has been implicated in inflammatory lung disease [3], and is often used as a measure of inflammation in cell culture research [4]. Cigarette smoke induces IL-6 in lung tissue [5] and levels relate to COPD disease severity [6]. Systemically, IL-6 induces skeletal muscle wasting
[7] and probably heightens exacerbation risk [8], both of which are major comorbidities in COPD.

Nuclear factor (NF)- $\kappa \mathrm{B}$ is an important transcriptional factor in the regulation of inflammatory genes in the airways of people with COPD [9]. Previous investigators have reported a reduced activation of NF- $\kappa \mathrm{B}$ in PBECs after exposure to CSE [10], while others have reported increased expression in bronchial biopsies obtained from smokers and COPD subjects [9]. Activated NF- $\mathrm{B}$ can have pro-apoptotic effects in certain cells, yet be protective of apoptosis in others [11]. CSE has been reported to induce apoptosis in PBECs [12] and primary nasal epithelial cells by some [13], but not by other investigators [10]. Furthermore, recent research using PBECs indicated that CSE induces necrosis rather than apoptosis [14].

\section{AFFILIATIONS}

*Belfast City Hospital, Belfast,

\# Mater Hospital, Belfast, and

"Queens University Belfast, Belfast, UK.

+Joint senior authors.

\section{CORRESPONDENCE}

J.S. Elborn

Centre for Infection and Immunity, Health Sciences Building, School of Medicine, Dentistry and Biomedical Sciences

Queens University Belfast

97 Lisburn Road

Belfast

BT9 7BL

UK

E-mail: s.elborn@qub.ac.uk

Received:

April 172012

Accepted after revision:

July 222012

First published online:

Aug 092012 
CSE provides a tool to explore the impact cigarette smoke has on PBEC cultures and facilitates our understanding of crucial intracellular signalling pathways. There is no consensus on the capacity for CSE to induce a pro-inflammatory response in epithelial cells. There is considerable diversity in the methods adopted to prepare CSE and no agreed gold standard. However, the weight of evidence would support that CSE has predominantly pro-inflammatory $[2,15,16]$ as opposed to immunosuppressive effects $[17,18]$ on bronchial epithelial cells. Although there is evidence that nasal epithelial cells can act as a satisfactory substitute for bronchial epithelial cells for certain end-points, we have recently shown that bronchial epithelial cells respond differently to nasal epithelial cells to CSE treatment [19].

In this study we investigated the responses of PBECs obtained from subjects with COPD, smokers with no airflow obstruction and nonsmoking control subjects to CSE treatment. To address this, we cultured cells at the air-liquid interface (ALI), which most closely resembles the in vivo environment, and stimulated cells with Pseudomonas aeruginosa lipopolysaccharide (LPS) as a surrogate for infection, with or without pretreatment with CSE. We aimed to determine the effects of CSE on inflammatory responses to $P$. aeruginosa LPS and levels of apoptosis in COPD epithelial cells compared to smokers without airflow obstruction and nonsmokers.

\section{METHODS}

\section{Study subjects}

16 subjects with a diagnosis of COPD according to the British Thoracic Society guidelines (13 of whom were current smokers, three ex-smokers), 10 smokers without airflow obstruction and nine nonsmokers were included. Subject demographics are detailed in table 1. All study subjects provided written informed consent to have a fibre-optic bronchoscopy, during which between four and six bronchial brushings obtained under direct visual guidance by means of a protected brush were obtained from the third generation bronchus. Brushings were placed in bronchial epithelium growth medium and transported to the laboratory for culture. Exclusion criteria included other significant pulmonary pathologies, including lung carcinoma. None of the patients had taken oral corticosteroids for at least 8 weeks prior to the study and no individual had a bronchodilator reversibility of greater than $10 \%$. Patients with COPD had no recent exacerbations and their current treatments are shown in table 1 . The 10 smokers without airflow obstruction required a bronchoscopy for clinical requirements such as persisting haemoptysis, but no abnormalities were evident during the procedure. The nine nonsmokers took part on a voluntary basis. This study was approved by the office for research ethics committees, Northern Ireland (REC: 09/NIR03/42).

\section{Cell culture}

PBECs were initially expanded in bronchial epithelial growth medium (BEGM; Promocell, Heidelberg, Germany) in purified bovine collagen coated (PureCol; Advanced Biomatrix, San Diego, CA, USA) T10 flasks and then T75 flasks for expansion with penicillin streptomycin antibiotics (Invitrogen, Grand Island, NY, USA) and Primocin (Invivogen, San Diego, CA, USA). Cells were confirmed to be epithelial in origin by randomly staining cultures by immunocytochemical staining for cytokeratin expression (data not shown). All experiments were performed in ALI cultures by seeding cells onto collagen coated Transwells (Corning Inc., Corning, NY, USA) at a seeding density of $1.5 \times 10^{5}$ cells per well and grown until cultures became confluent and developed tight junctions. At this stage the apical media was removed and the cell cultures fed basolaterally only on alternate days for 28 days.

\section{CSE}

CSE was prepared by a modification of the method of RICHTER et al. [15]. One commercial Marlboro Red cigarette $(0.8 \mathrm{mg}$ nicotine; $10 \mathrm{mg}$ tar; $10 \mathrm{mg}$ carbon monoxide) was combusted with a modified syringe-driven apparatus. The smoke was bubbled through $25 \mathrm{~mL}$ of media over $5 \mathrm{~min}$ by drawing 35$\mathrm{mL}$ volume of smoke every $15 \mathrm{~s}$. The resulting suspension was filtered through a $0.2 \mu \mathrm{m}$ pore-size filter to remove large particles and bacteria. This solution was regarded as " $100 \%$ CSE" and was freshly generated for each experiment, and subsequently serially diluted with culture medium to obtain a final $5 \%$ working concentration.

TABLE 1 Characteristics of the subject population

\begin{tabular}{lccc} 
& Nonsmokers & Smokers without airflow obstruction & COPD \\
\hline Subjects $\mathbf{n}$ & 9 & 10 & 16 \\
Age years & $51.5 \pm 4.7$ & $45.4 \pm 2.9$ & $66.7 \pm 3.3$ \\
Pack years & & $29.6 \pm 4.1$ & $31.1 \pm 7.2$ \\
Current cigarette consumption & & $17.6 \pm 2.1$ & $14.4 \pm 3.9$ \\
FEV 1 L & $2.89 \pm 0.21$ & $2.42 \pm 0.14$ & $1.56 \pm 0.24$ \\
FEV 1 \% pred & $97.3 \pm 6.2$ & $88.4 \pm 3.7$ & $56.1 \pm 3.2$ \\
FEV 1 /FVC $\%$ & $85.1 \pm 2.9$ & $82.4 \pm 3.4$ & $56.5 \pm 6.2$ \\
ICS treatment $\mathbf{n}$ & 0 & 0 & 15 \\
LABA treatment $\mathbf{n}$ & 0 & 0 & 15 \\
\hline
\end{tabular}

Data are presented as mean \pm SEM, unless otherwise stated. COPD: chronic obstructive pulmonary disease; FEV 1 : forced expiratory volume in $1 \mathrm{~s} ; \%$ pred: \% predicted FVC: forced vital capacity; ICS: inhaled corticosteroid; LABA: long-acting $\beta$-agonist. 


\section{Immunofluoresence}

Selected cultures were stained with a rabbit anti-E-cadherin primary antibody to demonstrate the presence of tight junctions. Separate cultures were exposed to both a 1:200 dilution of rabbit anti-MUC5AC primary antibody and a mouse anti-acetylated $\alpha$ tubulin antibody to demonstrate the presence of goblet cells and cilia, respectively. Images were captured and visualised using LAS AF (Leica, Wetzlar, Germany) acquisition software. Full details are provided in the online supplementary material.

\section{FACS}

Toll-like receptor (TLR)-4 was determined using fluorescenceactivated cell sorter (FACS) by staining permeabilised cells with phycoerthrin-conjugated anti-TLR-4 monoclonal antibodies (eBioscience, San Diego, CA, USA). The results obtained with specific antibodies were compared with those using isotype-matched control antibodies. Analysis of 10000 events was performed using an Epics XL flow cytometer (Beckman Coulter, UK Ltd, High Wycombe, UK).

Apoptosis was analysed using Annexin V and propidium iodide (PI) staining (eBioscience, UK). For experiments using CSE, cells were treated with $5 \%$ CSE for $24 \mathrm{~h}$ and the amount of apoptosis and/or necrosis was measured. Events which were positive for Annexin V, but negative for PI were considered to be early apoptotic, events positive for both Annexin V and PI late apoptotic, and events positive for PI alone necrotic. Full details are provided in the online supplementary material.

\section{TUNEL assay}

After treating PBECs with CSE for $24 \mathrm{~h}$, cultures were analysed for apoptosis using the Click-It TUNEL assay (Invitrogen, UK). Cells, after being fixed and permeabilised, were exposed to a reaction cocktail overnight at room temperature. Cells were then treated with a reaction buffer additive mixture for $30 \mathrm{~min}$, and cells mounted onto glass coverslips with mounting media and DAPI. 10 randomly selected high power fields at a final magnification of $\times 100$ were counted and the results expressed as the total number of apoptotic cells divided by the total number of cells per field. Full details are provided in the online supplementary material.

\section{Western blots}

Western blotting was used to determine phosphorylation of p38, ERK and JNK1 MAPK, cleaved caspase 3, IкB- $\alpha$ and phospho-NF- $\mathrm{B}$ after $4 \mathrm{~h}$ stimulation with $P$. aeruginosa LPS, with or without $5 \%$ CSE pretreatment for $24 \mathrm{~h}$. Full details are provided in the online supplementary material.

\section{ELISA}

Cytokine concentrations were measured from aliquots of basolateral and apical culture medium after appropriate stimulation as outlined in the results section using commercial IL-6 and IL-8 ELISA kits (R\&D Systems Europe, Abingdon, UK) according to the manufacturer's instructions. A chromogenic endotoxin quantification kit was used for the detection of bacterial endotoxins according to manufacturer's instructions (Thermoscientific, Cambridge, UK).

\section{TransAM NF- $\boldsymbol{k} B$ assay}

Nuclear extracts were prepared using a nuclear extraction kit from Active Motif (La Hulpe, Belgium) according to the manufacturer's instructions. Details are provided in the online supplementary material. The Active Motif Trans-AM NF-кB ELISA kit (Active Motif) was used to determine the levels of p65 in nuclear extracts. In brief, $2 \mu \mathrm{g}$ of nuclear extract, diluted to $20 \mu \mathrm{L}$, was added to the wells coated with oligonucleotides containing the NF- $\mathrm{KB}$ consensus binding site. The primary antibodies used to detect $\mathrm{NF}-\kappa \mathrm{B}$ recognise an epitope on $\mathrm{p} 65$ that is accessible only when NF- $\kappa \mathrm{B}$ is activated and bound to its target DNA. After the addition of secondary antibodies conjugated with HRP and substrate, absorbance was read at $450 \mathrm{~nm}$ (with a reference wavelength at $650 \mathrm{~nm}$ ). In order to monitor for specificity, competitive binding assays were performed. Wild-type or mutated consensus oligonucleotides were added to the wells containing immobilised oligonucleotides before the addition of nuclear extracts.

\section{Statistics}

Statistical analysis was performed using SPSS version 17.0 (SPSS Inc., Chicago, IL, USA). Data are presented as median values with interquartile ranges. Comparisons between groups were performed using the nonparametric Kruskal-Wallis test for multiple comparisons and the Mann-Whitney test for two groups. A p-value of less than 0.05 was considered significant.

\section{RESULTS}

\section{Cell culture}

Cells were successfully cultured in Transwells, developed tight junctions and had a cobblestone appearance when examined using a phase contrast light microscope. Mucus secretion was apparent after approximately 7 days in culture. After 14 days, there was an increase in the number of ciliated cells and after 28 days in culture a marked increase in their number was apparent (supplementary figure S1).

\section{CSE}

The optical density of a 5\% CSE concentration at $450 \mathrm{~nm}$ did not vary significantly when comparing a series of $5 \%$ CSE preparations (optical density $\sim 0.25$ ). Preparing CSE using a single cigarette in $25 \mathrm{~mL}$ of media produced a sufficiently consistent preparation. Previous investigators have reported similar findings [17]. Endotoxin was not detected in our 5\% CSE preparation.

\section{Soluble mediator release}

Constitutive and stimulated release of IL- 8 and IL- 6 was used to determine epithelial cell activation. Protein concentration was not significantly different from Transwells selected at random determined using a BCA assay (data not shown), and so soluble mediator release was expressed in $\mathrm{pg} \cdot \mathrm{mL}^{-1}$ rather than normalised to protein concentration. Concentrations of P. aeruginosa LPS (Sigma-Aldrich, Gillingham, UK) higher than $50 \mu \mathrm{g} \cdot \mathrm{mL}^{-1}$ were cytotoxic and lower concentrations released less IL-8. The release of both IL- 8 and IL- 6 after stimulation with $50 \mu \mathrm{g} \cdot \mathrm{mL}^{-1}$ P. aeruginosa LPS from cultures obtained from control subjects and smokers without airflow obstruction was heightened after pretreatment with CSE for $24 \mathrm{~h}$. In the COPD cultures, 5\% CSE pretreatment mitigated the stimulatory effects of $p$. aeruginosa LPS (fig. 1).

In separate experiments, cells obtained from a nonsmoking subjects were incubated with various concentrations of CSE (1$50 \%$ ) for $24 \mathrm{~h}$. CSE stimulated the cultures to release IL-8 up to 


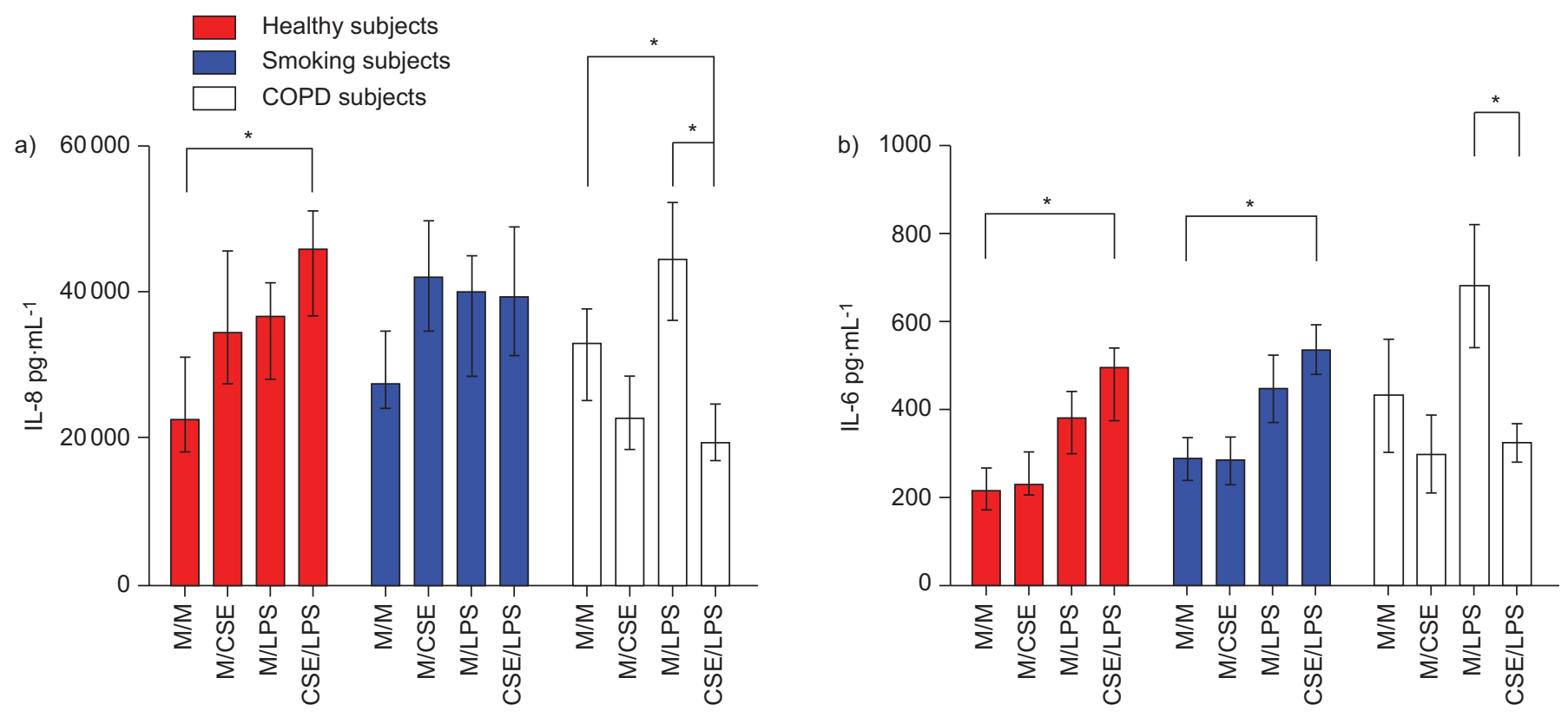

FIGURE 1. a) Interleukin (IL)-8 and b) IL-6 release from apical supernatants in primary bronchial epithelial cell (PBEC) air-liquid interface (ALI) cultures from chronic obstructive pulmonary disease (COPD) subjects, smoking subjects and healthy control subjects after treatment with Pseudomonas aeruginosa lipopolysaccharide (LPS) $\left(50 \mu \mathrm{g} \cdot \mathrm{mL}^{-1}\right)$ for $24 \mathrm{~h}$, with or without pretreatment with $5 \%$ cigarette smoke extract (CSE) for $24 \mathrm{~h}$. Well differentiated PBEC ALI cultures were treated with P. aeruginosa LPS $\left(50 \mu \mathrm{g} \cdot \mathrm{mL}^{-1}\right)$ for $24 \mathrm{~h}$, with or without pretreatment with $5 \%$ CSE (or vehicle) for $24 \mathrm{~h}$ ( $\mathrm{n}=7$ for each group). Apical supernatants were collected and assessed for IL-8/L-6 by ELISA. Data are displayed as medians and interquartile ranges. M/M: media (24 h) and media (24 h); M/CSE: media (24 h) and $5 \%$ CSE (24 h); M/LPS: media (24 h) and LPS $50 \mu \mathrm{g} \cdot \mathrm{ml}^{-1}(24 \mathrm{~h}) ; \mathrm{CSE} / \mathrm{LPS}: 5 \%$ CSE $(24 \mathrm{~h})$ and LPS $50 \mu \mathrm{g} \cdot \mathrm{ml}^{-1}(24 \mathrm{~h}) .{ }^{*}: \mathrm{p}<0.05$.

a $5 \%$ concentration, subsequently falling at higher concentrations due to cytotoxicity (fig. 2).

\section{CSE induces apoptosis in primary bronchial epithelial cell cultures}

Apoptosis was confirmed using the TUNEL assay. For the representative image (fig. 3), the number of apoptotic cells determined by this technique was $4 \%$ for the healthy subject and $19 \%$ for the corresponding patient with COPD after cells were exposed to CSE for $24 \mathrm{~h}$.

Using the FACS technique, also after treatment with 5\% CSE for $24 \mathrm{~h}$ in the COPD cells: $51 \%$ of cells remained viable (Annexin V and PI negative); $19 \%$ were undergoing early apoptosis; $26 \%$ undergoing late apoptosis; and $4 \%$ were necrotic (fig. 4). In separate experiments, cells from each of the three study groups (again after treatment with CSE for $24 \mathrm{~h}$ ) were stained with
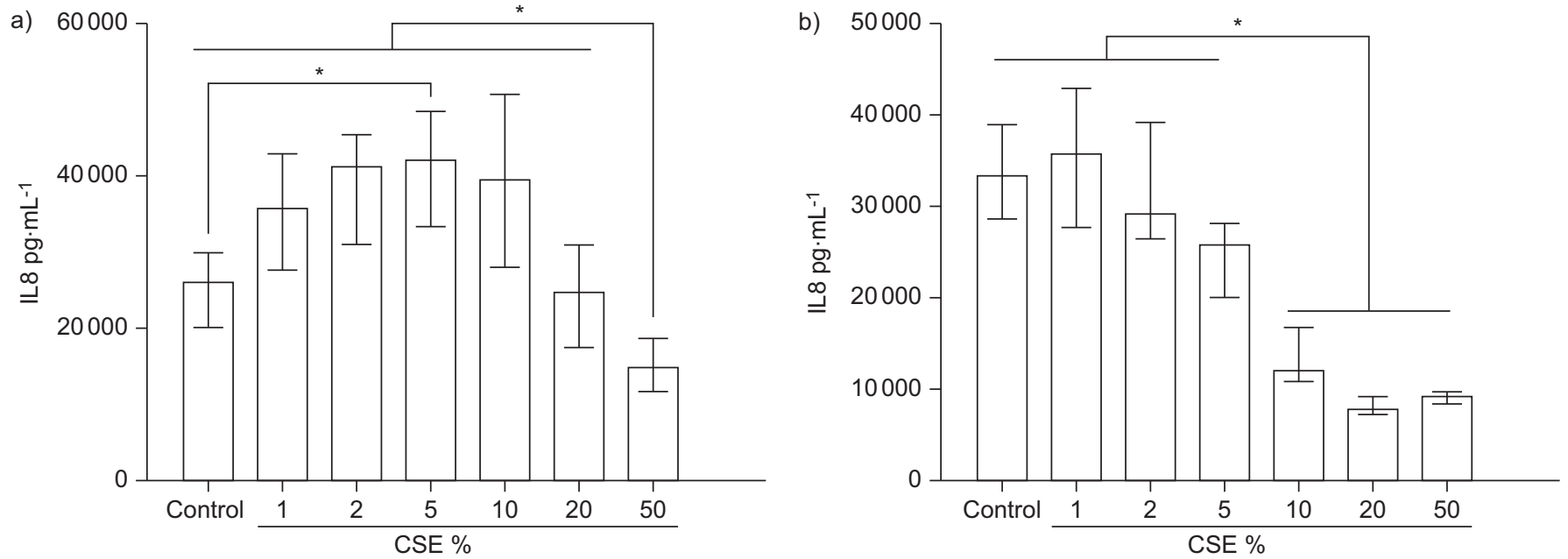

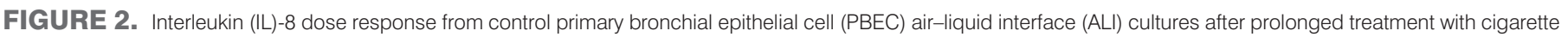

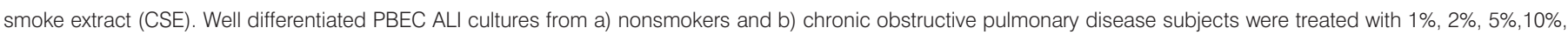

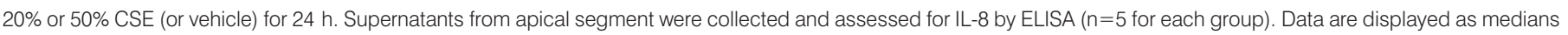
and interquartile ranges. ${ }^{*}: p<0.05$. 

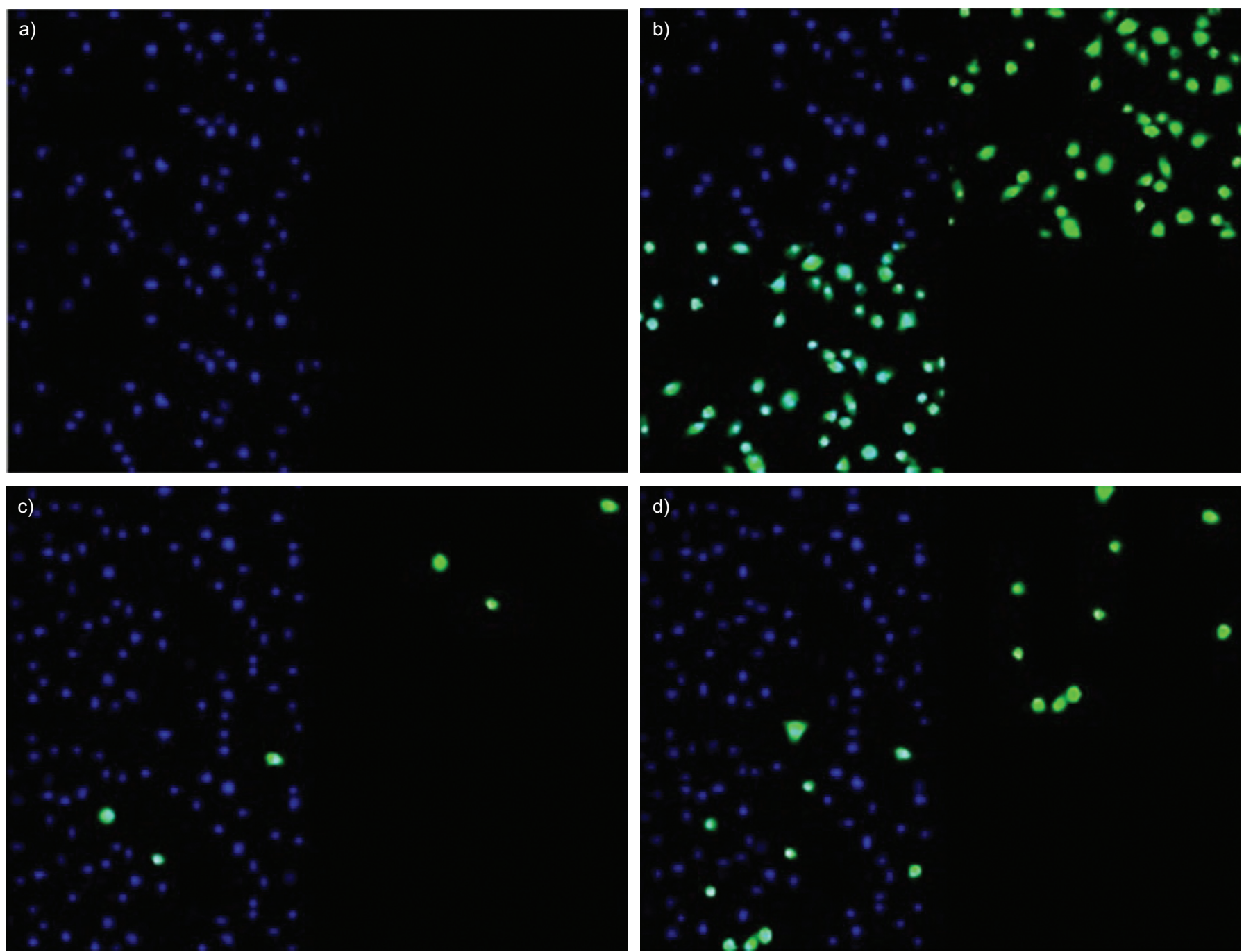

FIGURE 3. Effect of $5 \%$ cigarette smoke extract (CSE) on apoptosis in primary bronchial epithelial cell cultures from healthy subjects and smokers from air-liquid interface cultures determined using the TUNEL assay. Representative images of bronchial epithelial cells from nonsmoking subjects or smokers without airflow obstruction were grown on coverslips and treated with a) PBS or b) DNase I solution for the negative and positive control, respectively. Cultures obtained from c) a nonsmoking subject and from d) a chronic obstructive pulmonary disease subject were treated with 5\% CSE for $24 \mathrm{~h}$ and the number of apoptotic cells counted using the Click-iT reaction according to manufacturer's instructions.

Annexin $\mathrm{V}$ to determine any differences in the levels of apoptosis between the groups. CSE induced the highest percentage of apoptosis from the COPD cultures, with the least amount from the nonsmokers. Representative dot plots are shown in figure 5.

\section{CSE cleaves full length caspase-3 in PBEC cultures}

Using an antibody against the cleaved fragment of caspase-3 $(17 \mathrm{kDa})$, we demonstrated by Western blotting that treatment with $P$. aeruginosa LPS and CSE increased the amount of active caspase-3, whereas P. aeruginosa LPS alone did not. Furthermore, the COPD cultures had the greatest amount of cleaved caspase- 3 after $P$. aeruginosa LPS and CSE stimulation. Cleaved caspase to actin ratio, as determined by densitometry and a representative blot, are shown in figure 6.

In separate experiments, COPD cultures were treated with increasing concentrations of $P$. aeruginosa LPS $\left(0-50 \mu \mathrm{g} \cdot \mathrm{mL}^{-1}\right)$ and CSE (5-50\%) and caspase-3 was determined using an antibody against full length caspase $(35 \mathrm{kDa})$ and its cleaved fragment
(17 kDa). P. aeruginosa LPS alone did not cleave full length caspase, but there was definite cleavage using a 5\% CSE and increasingly so with higher CSE concentrations (supplementary figure S2).

\section{CSE reduces TLR-4 expression in COPD cell cultures}

To examine whether CSE regulates the expression of TLR-4, PBEC cultures were treated with $P$. aeruginosa $\mathrm{LPS}\left(50 \mu \mathrm{g} \cdot \mathrm{mL}^{-1}\right)$, with or without pretreatment with $5 \%$ CSE for $24 \mathrm{~h}$. Although there was no change in the absolute values for mean fluorescence intensity for TLR-4 in the cultures from control subjects after CSE treatment, CSE treatment reduced the mean fluorescence intensity for TLR-4 in the COPD cultures. Representative histograms are shown in figure 7.

\section{CSE reduces MAPK activation and NF- $\kappa B$ in COPD cell cultures}

Western blotting showed a reduced phosphorylation of p38, JNK and ERK MAPK in the COPD cultures after treatment 

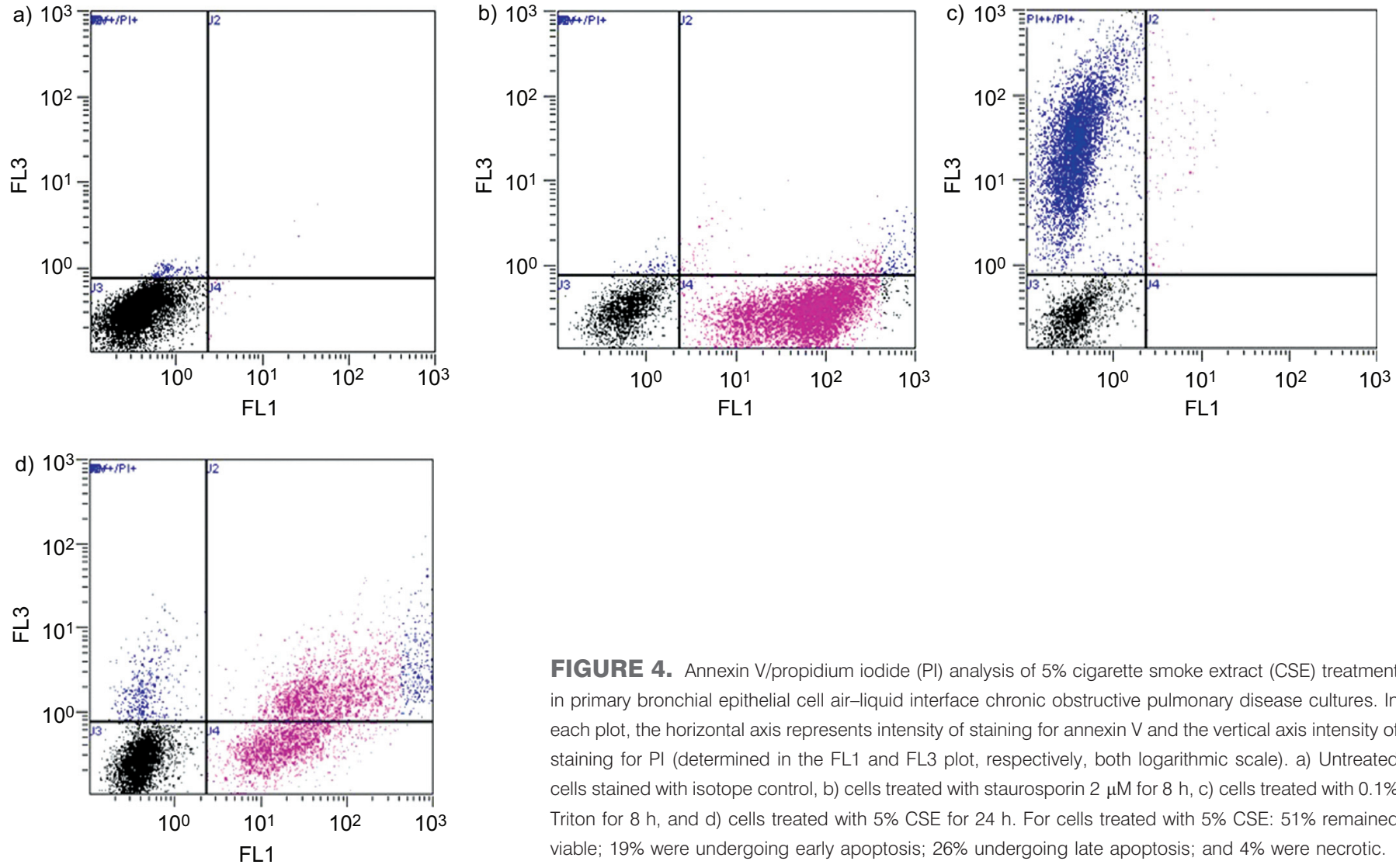

FIGURE 4. Annexin V/propidium iodide (PI) analysis of $5 \%$ cigarette smoke extract (CSE) treatment in primary bronchial epithelial cell air-liquid interface chronic obstructive pulmonary disease cultures. In each plot, the horizontal axis represents intensity of staining for annexin $\vee$ and the vertical axis intensity of staining for PI (determined in the FL1 and FL3 plot, respectively, both logarithmic scale). a) Untreated cells stained with isotope control, b) cells treated with staurosporin $2 \mu \mathrm{M}$ for $8 \mathrm{~h}, \mathrm{c}$ ) cells treated with $0.1 \%$ Triton for $8 \mathrm{~h}$, and d) cells treated with 5\% CSE for $24 \mathrm{~h}$. For cells treated with 5\% CSE: 51\% remained viable; $19 \%$ were undergoing early apoptosis; $26 \%$ undergoing late apoptosis; and $4 \%$ were necrotic.

with 5\% CSE for $24 \mathrm{~h}$. All MAPK were activated, even without stimulation, with no further significant increase after P. aeruginosa LPS stimulation (lanes 1-4 in all blots; supplementary figure S3-S5). However, levels of phosphorylated p38, ERK and JNK were reduced with $24 \mathrm{~h} \mathrm{5 \%}$ CSE pretreatment and $P$. aeruginosa LPS stimulation in the COPD cultures (lanes 5-8 in all blots; supplementary figure S3-S5). The reduced activation of MAPK was not evident in the control cultures. The reduced activation was not as pronounced for p38 compared with JNK or ERK, and so this was also measured using FACS which confirmed a reduced mean fluorescence intensity for phosphop38 after 5\% CSE treatment (supplementary figure S3).
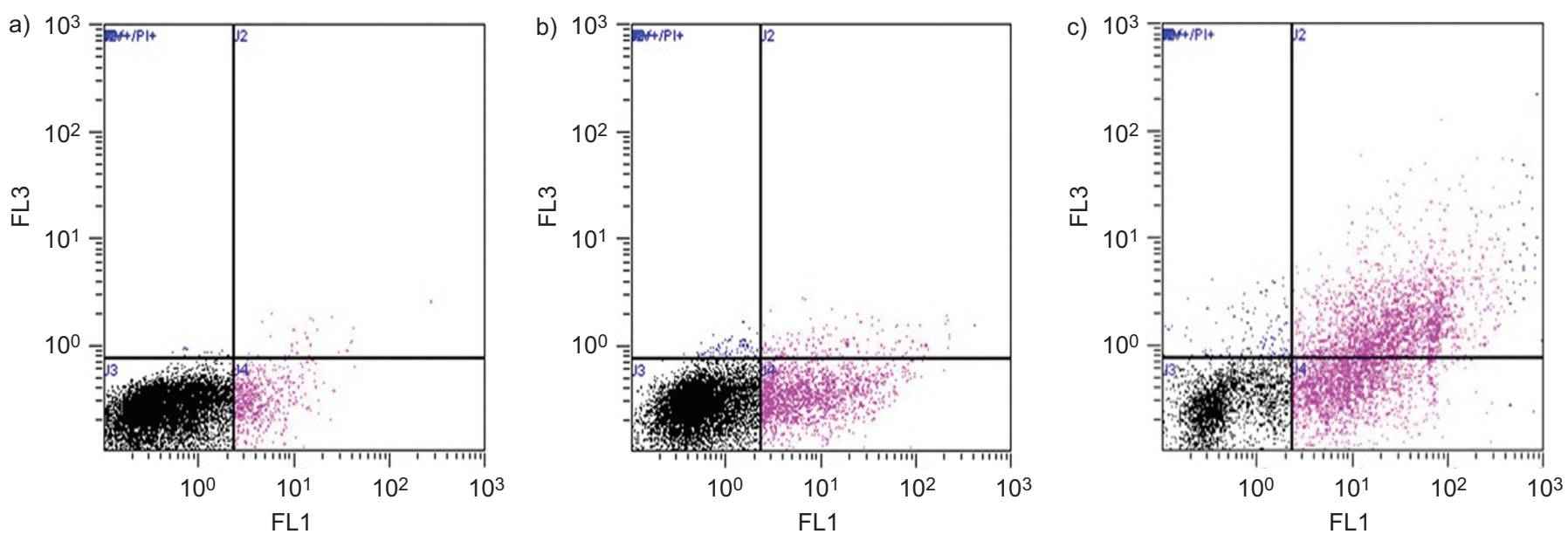

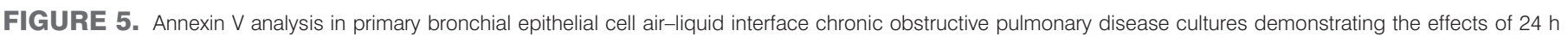

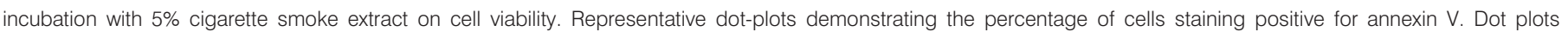

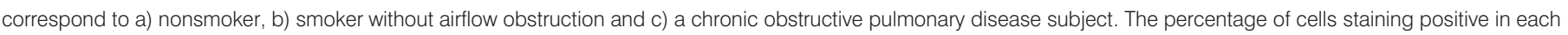
quadrant are indicated in table 2. 
TABLE 2 Effect of $24 \mathrm{~h}$ incubation with 5\% cigarette smoke extract on cell viability

\begin{tabular}{lcccc} 
& Viable $\%$ & Early Apoptosis \% & Late Apoptosis \% & Necrotic \% \\
\hline Nonsmoker & 94 & 5 & 1 & 0 \\
Smoker & 82 & 16 & 2 & 0 \\
COPD & 49 & 20 & 22 & 9 \\
\hline
\end{tabular}

COPD: chronic obstructive pulmonary disease.

There was a significant increase phospho-NF- $\mathrm{B}$ protein level from whole cell lysates after stimulation with $50 \mu \mathrm{g} \cdot \mathrm{mL}^{-1}$ $P$. aeruginosa LPS for all three groups. This was further heightened in the nonsmoker and smokers without airflow obstruction groups after pretreatment with 5\% CSE. In contrast, in the COPD cultures, pretreatment with 5\% CSE reduced

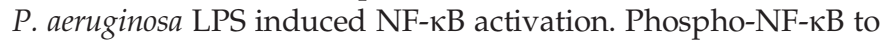
actin ratio as determined by densitometry and a representative blot are shown in figure 8. Levels of I $\mathrm{I} B-\alpha$ decreased as phospho-NF- $\kappa B$ increased. Repeating the experiments and determining levels of p65 NF- $\mathrm{kB}$ from nuclear extracts using an ELISA based method demonstrated similar results (fig. 9).
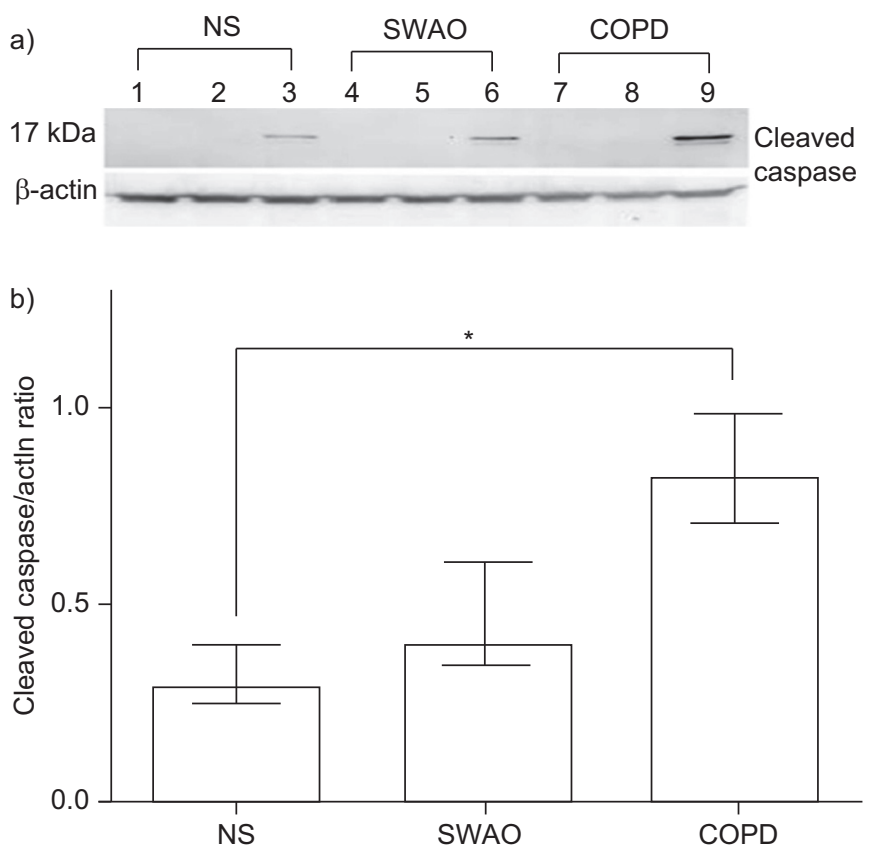

FIGURE 6. Effect of Pseudomonas aeruginosa lipopolysaccharide (LPS) and $5 \%$ cigarette smoke extract (CSE) on caspase-3 activation in primary bronchial epithelial cell air-liquid interface (ALI) cultures determined by Western blotting. a) Lanes 1-3 represent treatment of ALI cultures from a nonsmoking (NS) subject with media, $50 \mu \mathrm{g} \cdot \mathrm{mL}^{-1} \mathrm{P}$. aeruginosa LPS $(4 \mathrm{~h})$, and $5 \%$ CSE $(24 \mathrm{~h})$ followed by $50 \mu \mathrm{g} \cdot \mathrm{mL}^{-1} \mathrm{P}$. aeruginosa LPS $(4 \mathrm{~h})$, respectively. Lanes $4-6$ represent equivalent stimulation in ALI cultures obtained from a smoker without airflow obstruction (SWAO) and lanes 7-9 ALI cultures from a subject with chronic obstructive pulmonary disease. The upper blot represents the large fragment of caspase-3 resulting from cleavage $(17 \mathrm{kDa})$ and the lower $\beta$-actin. b) Cleaved caspase:actin ratio following exposure to $5 \%$ CSE ( $n=5$ for each group). Data are displayed as medians and interquartile ranges. *: $p<0.05$.

\section{DISCUSSION}

In this series of experiments we have demonstrated that the stimulated release of IL- 6 and IL-8 from cultures obtained from controls and smokers without airflow obstruction is amplified by $5 \%$ CSE pretreatment, but this was not apparent in the COPD group. Our results also show cellular immunosuppressive effects of 5\% CSE in the COPD cultures. These findings suggest that COPD PBECs are more susceptible to the immunosuppressive effects of CSE which may explain, in part, the increased susceptibility of this particular group to respiratory infections.

In our experiments, 5\% CSE reduced the expression of TLR-4. Similar findings were recently reported using a human bronchial epithelial cell line, where CSE treatment led to a downregulation of TLR-4 expression, which was associated with a corresponding increase in the release of IL-8. Internalisation of the receptor was proposed as the mechanism, as there was a parallel increase in the expression of TLR-4 in permeabilised cells [4]. However, the reported effects of CSE on TLR-4 expression are not entirely consistent. Although there was a dose dependent downregulation of TLR-4 mRNA and protein expression in A549 cells, after stimulation with CSE [20], a publication the following year suggested that CSE increases TLR-4 expression in BEAS-2B cells [21]. CSE can also affect other important receptors, such as the RIG-1 receptors, by downregulating their surface expression [22]. Therefore, although our data indicate that CSE downregulates TLR-4 expression in COPD PBECs, we cannot be absolutely confident that this is the sole mechanism of its immunosuppressive effects. It is conceivable that CSE also affects other receptors, which may affect cell stimulation independent of TLR-4.

Activation of NF- $\mathrm{KB}$ regulates gene expression, promotes cell survival, and serves to protect cells from apoptosis in murine B cells and in a number of cell lines [23, 24]. Furthermore, activation of NF- $\mathrm{KB}$ through a TLR-dependent activation by hyaluronan in airway epithelial cells has also been shown to be protective of apoptosis [25]. Interestingly, as the absolute amount of TLR-4 was reduced in the COPD epithelial cell cultures after treatment with CSE, which was associated with a reduction in the activation of all MAPKs, this is a potential mechanism for apoptosis in these particular cultures to CSE treatment. However, other data do not support this concept, as treating PBECs obtained from healthy subjects with CSE for $48 \mathrm{~h}$ led to inhibition of NF- $\mathrm{BB}$ activation without any evidence of apoptosis, necrosis or caspase-3 activation [10].

As well as differences in soluble mediator release, we have demonstrated differences in phosphorylated NF- $\mathrm{KB}$ in our study groups. Although treating PBECs obtained from healthy 
a)

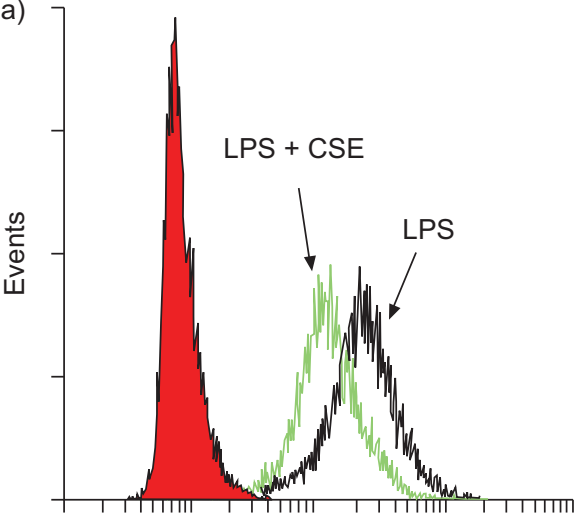

Fluorescence intensity

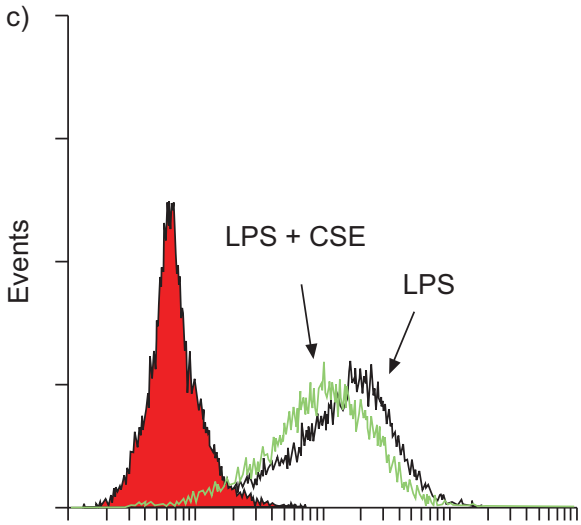

Fluorescence intensity

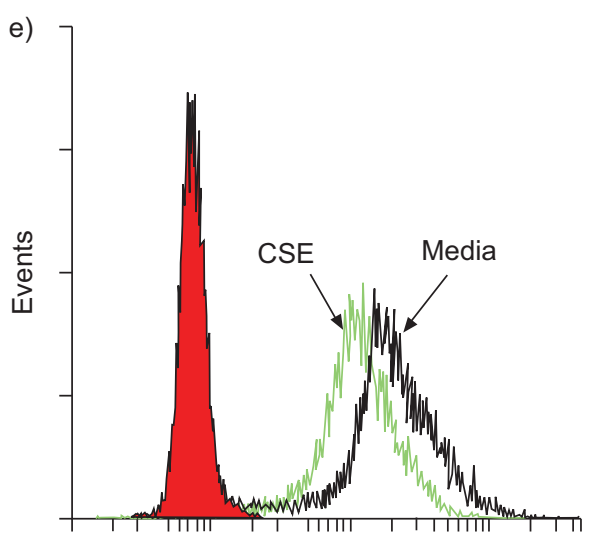

Fluorescence intensity

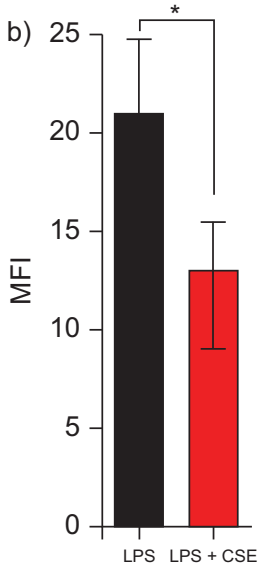

d) 20
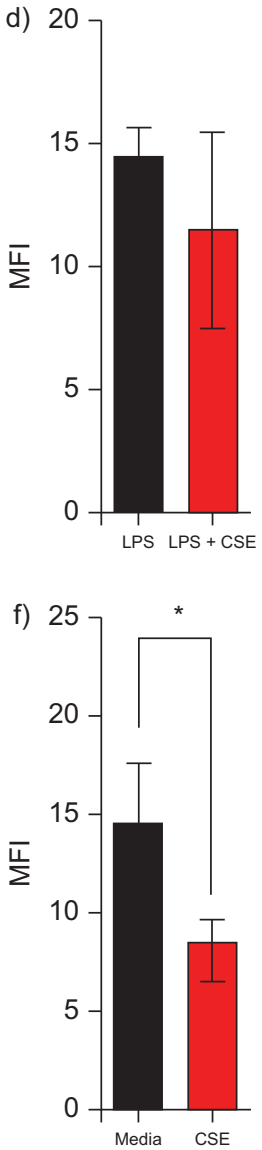

FIGURE 7. Representative histogram of amount of intracellular Toll-like receptor (TLR)-4 in primary bronchial epithelial cell (PBEC) air-liquid interface (ALI) cultures after stimulation with Pseudomonas aeruginosa lipopolysaccharide (LPS) with or without $5 \%$ cigarette smoke extract (CSE) pretreatment. Well differentiated PBEC ALI cultures from a) and b) a subject with chronic obstructive pulmonary disease (COPD), and cultures from c) and d) a smoker without airflow obstruction were incubated with 5\% CSE for $24 \mathrm{~h}$ and then stimulated with $P$. aeruginosa LPS $\left(50 \mu \mathrm{g} \cdot \mathrm{mL}^{-1}\right)$ for $4 \mathrm{~h}$. e) and f) Separate PBEC ALI cultures from a COPD subject were treated either with or without a $5 \%$ CSE alone for $24 \mathrm{~h}$. Cells were then fixed, permeabilised and stained with a phycoerthrin-conjugated antibody against TLR-4 or equivalent isotype-matched control. Mean fluorescence intensity (MFI) is shown ( $n=4$ for each group). Data are displayed as medians and interquartile ranges. ${ }^{*}: p<0.05$.
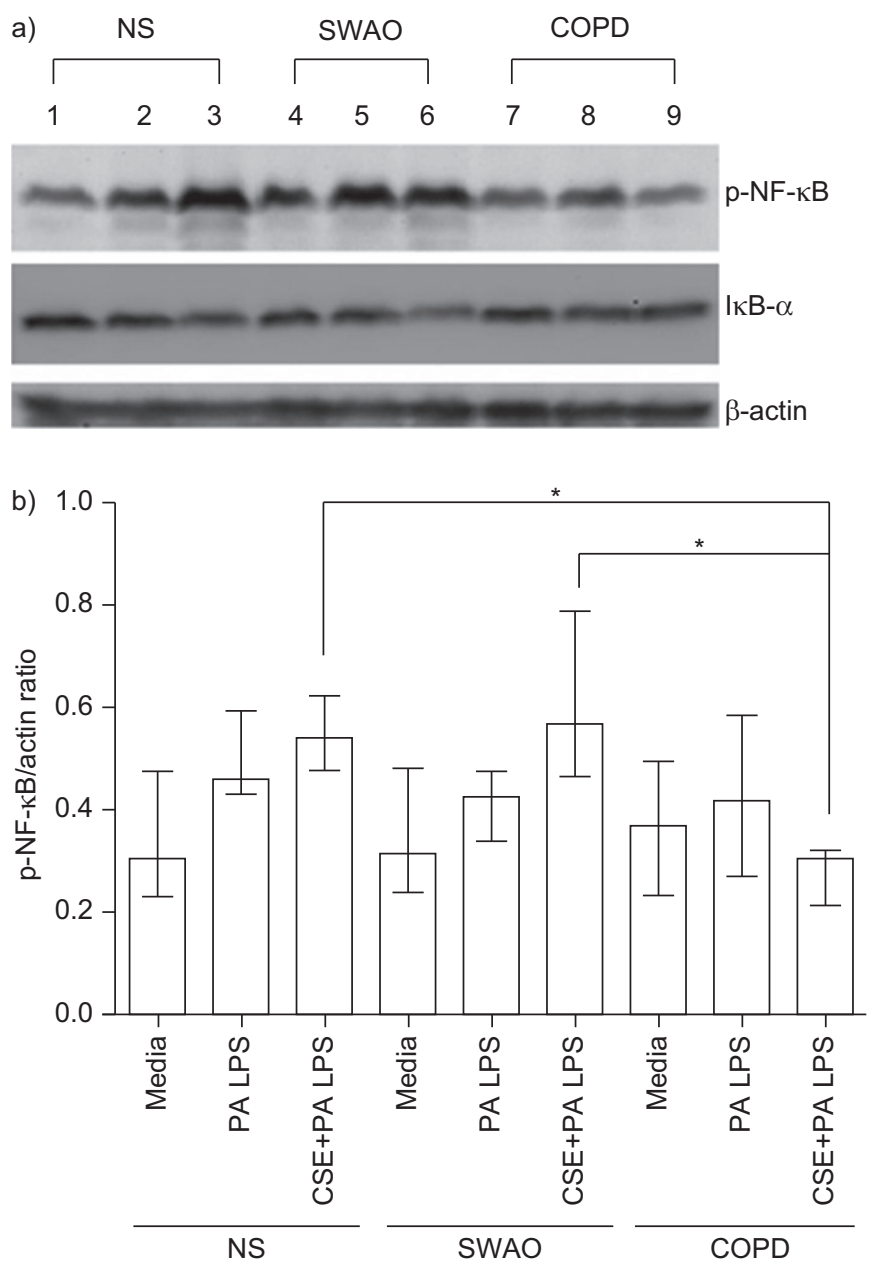

FIGURE 8. Effect of lipopolysaccharide (LPS) with or without $5 \%$ cigarette smoke extract (CSE) on nuclear factor (NF)-kB activation in primary bronchial epithelial cell (PBEC) air-liquid interface (ALI) cultures determined by Western blotting. a) Western blot of $\mathrm{p} 65 \mathrm{NF}-\mathrm{kB}$ and $1 \mathrm{kB}-\alpha$ protein expression (with $\beta$-actin loading control) in PBEC ALI cultures of control nonsmokers (NS), smokers without airflow obstruction (SWAO) and chronic obstructive pulmonary disease (COPD) patients after treatment with $50 \mu \mathrm{g} \cdot \mathrm{mL}^{-1}$ Pseudomonas aeruginosa LPS for $4 \mathrm{~h}$ or $5 \%$ CSE treatment for $24 \mathrm{~h}$ followed by treatment with $50 \mu \mathrm{g} \cdot \mathrm{mL}^{-1} \mathrm{P}$. aeruginosa LPS for $4 \mathrm{~h}$. Lanes 1-3 represent treatment of nonsmoker PBEC's with media alone, $50 \mu \mathrm{g} \cdot \mathrm{mL}^{-1} \mathrm{P}$. aeruginosa LPS (4 h) and $50 \mu \mathrm{g} \cdot \mathrm{mL}^{-1} \mathrm{P}$. aeruginosa LPS (4 h) with $5 \%$ CSE pretreatment $(24 \mathrm{~h})$. Lanes $4-6$ represent equivalent treatments in an ALI culture from a smoker without airflow obstruction and 7-9 in those from a COPD subject. b) Phospho-NF-kB to actin ratio ( $n=5$ for each group). Data are displayed as medians and interquartile ranges. *: $p<0.05$.

control subjects and smokers without airway disease with $P$. aeruginosa LPS increased the amount of phosphorylated NF- $\mathrm{kB}$, this was not the case for the COPD ALI cultures. Furthermore, the COPD ALI cultures had a reduced activation of all MAPK after treatment with 5\% CSE. The latter observation was demonstrated in parallel with a reduced expression of TLR-4 and a diminished expression of the transcription factor, phosphorylated NF-кB.

The use of CSE for in vitro studies has been criticised as an unsatisfactory model for the long term, low concentration smoke which smokers are exposed to [26]. Concentrations of 


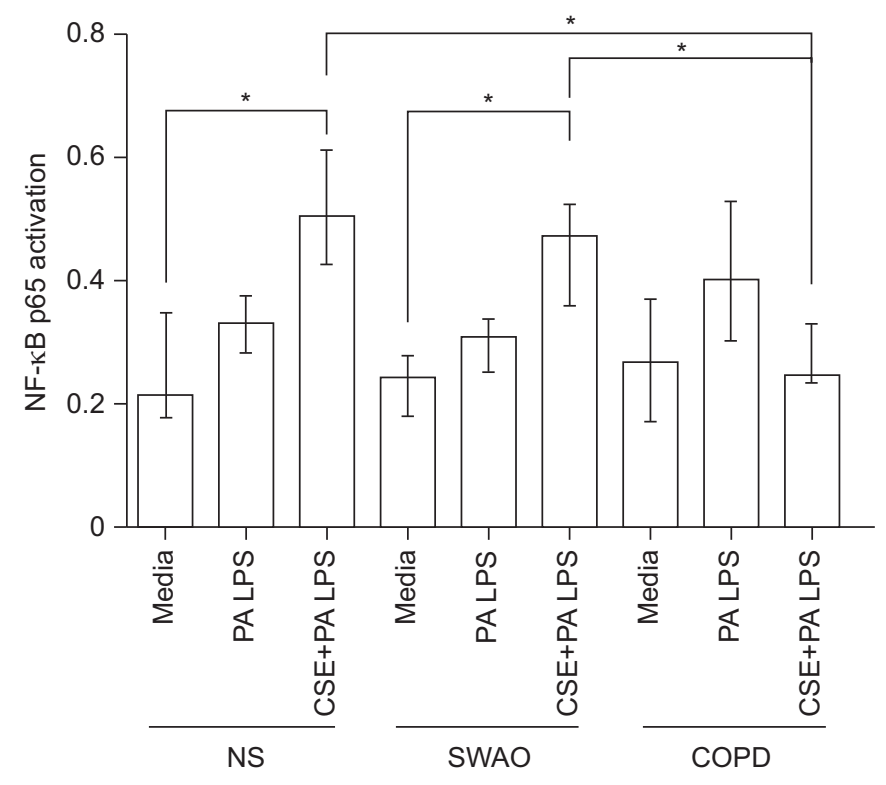

FIGURE 9. Effect of lipopolysaccharide (LPS) with or without $5 \%$ cigarette smoke extract (CSE) on nuclear factor (NF)-kB activation in primary bronchial epithelial cell (PBEC) air-liquid interface (ALI) cultures determined using the TransAM NF-kB kit. Nuclear extracts from PBEC ALI cultures of control nonsmokers (NS), smokers without airflow obstruction (SWAO) and chronic obstructive pulmonary disease (COPD) patients after treatment with $50 \mu \mathrm{g} \cdot \mathrm{mL}^{-1}$ Pseudomonas aeruginosa LPS for $4 \mathrm{~h}$ or $5 \%$ CSE treatment for $24 \mathrm{~h}$ followed by treatment with $50 \mu \mathrm{g} \cdot \mathrm{mL}^{-1} \mathrm{P}$. aeruginosa LPS for $4 \mathrm{~h}$ were assayed for NF-KB p65 activation using the TransAM NF$\kappa \mathrm{B}$ p65 Kit. Results shown are from wells assayed in duplicate.

CSE used can range from 100\% CSE for $15 \mathrm{~min}$ [17] to 1\% CSE for $24 \mathrm{~h}$ (in those studies which use a single cigarette to prepare the initial "100\%" stock CSE) [27]. Other investigators have prepared CSE using multiple cigarettes ranging from two cigarettes up to as many as five [13,28]. Many of these studies assessed soluble mediator release and a single time point, but two studies highlight that caution is required as CSE can delay LPS-induced release of IL-8 and granulocyte-macrophage colony stimulating factor in primary epithelial cells, but yet it was not abolished completely at later time points [17, 18]. Therefore, if earlier time points were used in isolation, the findings would misleadingly suggest that CSE was solely immunosuppressive. There was unacceptable cytotoxicity at concentrations of CSE greater than 5\% (supplementary figure S2), so we opted to use this preparation of CSE to study soluble mediator release from the ALI cultures.

The majority of the subjects with COPD used in this study were current smokers. Our data suggest that the increase in apoptosis, and the increase in caspase-3 activation relative to smokers without airflow obstruction and control subjects in response to CSE exposure was not related to cigarette smoking per se. It appears that the development of COPD itself further contributes to the increased susceptibility of the bronchial epithelium to the cytotoxic effects of CSE. Alternatively the epithelial cells from these individuals may be intrinsically more vulnerable to apoptosis.

Caspase activation has not consistently been shown to be important in CSE induced apoptosis [29], and in fact cell death can occur by mechanisms which are independent of caspase activity [30]. It is feasible that CSE alters the pro- and antiapoptotic factors which are important players in this increasingly complicated cell death pathway. CSE alters levels of phosphorylated NF-KB in PBEC ALI cultures, and our data suggest that a heightened NF- $\mathrm{KB}$ activation may be protective of apoptosis in this context.

Although it could be argued that the process of cell culturing may alter the epithelial phenotype through the multiple cycles of proliferation involved in achieving ALI cultures, recent research suggests this is unlikely to be the case, at least for primary nasal epithelial cells [31]. In addition, there are varied methods used to obtain and establish airway epithelial cell cultures, each with their own merits and limitations. Furthermore, the airways of individuals with COPD may not be sterile. We have discussed these important aspects in the online supplementary material.

To conclude, although airway epithelial cells from patients with COPD exhibit a constitutional pro-inflammatory phenotype, these cells have a diminished inflammatory response to CSE compared to control subjects. Furthermore, these cells have an increased susceptibility to undergo apoptosis. It is not clear if this abnormality results from a change to the cells rendered by the disease process, or whether these particular cells are fundamentally more vulnerable to cell death. Regardless of which explanation holds true, this particular abnormality at least suggests that therapies aimed at reducing the amount of apoptosis may be beneficial. The development inhibitors of apoptosis are realistic ambitions. There are already broad spectrum inhibitors of apoptosis in clinical trials for the treatment of liver disease and they represent the first in their class of agents [32]. Furthermore, this data emphasises the importance of smoking cessation in COPD subjects in order to minimise loss of epithelial cells and to maintain integrity of the bronchial epithelium. Our findings support the notion that cigarette smoke reduces innate lung defence and has the capacity to increase susceptibility to respiratory infections.

\section{STATEMENT OF INTEREST}

None declared.

\section{REFERENCES}

1 Barnes PJ. Mediators of chronic obstructive pulmonary disease. Pharmacol Rev 2004; 56: 515-548.

2 Kode A, Yang SR, Rahman I. Differential effects of cigarette smoke on oxidative stress and proinflammatory cytokine release in primary human airway epithelial cells and in a variety of transformed alveolar epithelial cells. Respir Res 2006; 7: 132.

3 Pease JE, Sabroe I. The role of interleukin- 8 and its receptors in inflammatory lung disease: implications for therapy. Am J Respir Med 2002; 1: 19-25.

4 Mortaz E, Henricks PA, Kraneveld AD, et al. Cigarette smoke induces the release of CXCL-8 from human bronchial epithelial cells via TLRs and induction of the inflammasome. Biochim Biophys Acta 2011; 1812: 1104-1110.

5 Vlahos R, Bozinovski S, Jones JE, et al. Differential protease, innate immunity, and NF- $\kappa B$ induction profiles during lung inflammation induced by subchronic cigarette smoke exposure in mice. Am J Physiol Lung Cell Mol Physiol 2006; 290: L931-L945. 
6 Broekhuizen R, Wouters EF, Creutzberg EC, et al. Raised CRP levels mark metabolic and functional impairment in advanced COPD. Thorax 2006; 61: 17-22.

7 Hansen MJ, Gualano RC, Bozinovski S, et al. Therapeutic prospects to treat skeletal muscle wasting in COPD (chronic obstructive lung disease). Pharmacol Ther 2006; 109: 162-172.

8 Yende S, Tuomanen EI, Wunderink R, et al. Preinfection systemic inflammatory markers and risk of hospitalization due to pneumonia. Am J Respir Crit Care Med 2005; 172: 1440-1446.

9 Di Stefano A, Caramori G, Oates T, et al. Increased expression of nuclear factor- $\mathrm{\kappa B}$ in bronchial biopsies from smokers and patients with COPD. Eur Respir J 2002; 20: 556-563.

10 Manzel LJ, Shi L, O'Shaughnessy PT, et al. Inhibition by cigarette smoke of nuclear factor- $\mathrm{KB}$-dependent response to bacteria in the airway. Am J Respir Cell Mol Biol 2011; 44: 155-165.

11 Shishodia S, Aggarwal BB. Nuclear factor- $\mathrm{\kappa B}$ activation: a question of life or death. J Biochem Mol Biol 2002; 35: 28-40.

12 Togo S, Sugiura H, Nelson A, et al. Hepatic growth factor (HGF) inhibits cigarette smoke extract induced apoptosis in human bronchial epithelial cells. Exp Cell Res 2010; 316: 3501-3511.

13 Lan MY, Ho CY, Lee TC, et al. Cigarette smoke extract induces cytotoxicity on human nasal epithelial cells. Am J Rhinol 2007; 21: 218-223.

14 Groskreutz DJ, Monick MM, Babor EC, et al. Cigarette smoke alters respiratory syncytial virus-induced apoptosis and replication. $A m$ J Respir Cell Mol Biol 2009; 41: 189-198.

15 Richter A, O'Donnell RA, Powell RM, et al. Autocrine ligands for the epidermal growth factor receptor mediate interleukin-8 release from bronchial epithelial cells in response to cigarette smoke. Am J Respir Cell Mol Biol 2002; 27: 85-90.

16 Mio $\mathrm{T}$, Romberger $\mathrm{DJ}$, Thompson $\mathrm{AB}$, et al. Cigarette smoke induces interleukin-8 release from human bronchial epithelial cells. Am J Respir Crit Care Med 1997; 155: 1770-1776.

17 Laan M, Bozinovski S, Anderson GP. Cigarette smoke inhibits lipopolysaccharide-induced production of inflammatory cytokines by suppressing the activation of activator protein- 1 in bronchial epithelial cells. J Immunol 2004; 173: 4164-4170.

$18 \mathrm{Li} \mathrm{W}, \mathrm{Xu} \mathrm{YJ}$, Shen HH. Effect of cigarette smoke extract on lipopolysaccharide-activated mitogen-activated protein kinase signal transduction pathway in cultured cells. Chin Med J (Engl) 2007; 120: 1075-1081.
19 Comer DM, Elborn JS, Ennis M. Comparison of nasal and bronchial epithelial cells obtained from patients with COPD. PLOS ONE 2012; 7: e32924.

20 MacRedmond RE, Greene CM, Dorscheid DR, et al. Epithelial expression of TLR4 is modulated in COPD and by steroids, salmeterol and cigarette smoke. Respir Res 2007; 8: 84.

21 Pace E, Ferraro M, Siena L, et al. Cigarette smoke increases Tolllike receptor 4 and modifies lipopolysaccharide-mediated responses in airway epithelial cells. Immunology 2008; 124: 401-411.

$22 \mathrm{Wu}$ W, Patel KB, Booth JL, et al. Cigarette smoke extract suppresses the RIG-I-initiated innate immune response to influenza virus in the human lung. Am J Physiol Lung Cell Mol Physiol 2011; 300: L821-L830.

$23 \mathrm{Wu} \mathrm{M}$, Lee $\mathrm{H}$, Bellas RE, et al. Inhibition of NF- $\mathrm{KB} /$ Rel induces apoptosis of murine B cells. EMBO J 1996; 15: 4682-4690.

24 Foo SY, Nolan GP. NF- $\mathrm{KB}$ to the rescue: RELs, apoptosis and cellular transformation. Trends Genet 1999; 15: 229-235.

25 Jiang D, Liang J, Fan J, et al. Regulation of lung injury and repair by Toll-like receptors and hyaluronan. Nat Med 2005; 11: 1173-1179.

26 Shapiro SD. Smoke gets in your cells. Am J Respir Cell Mol Biol 2004; 31: 481-482.

27 Witherden IR, Vanden Bon EJ, Goldstraw P, et al. Primary human alveolar type II epithelial cell chemokine release: effects of cigarette smoke and neutrophil elastase. Am J Respir Cell Mol Biol 2004; 30: 500-509.

28 Wang JH, Kim H, Jang YJ. Cigarette smoke extract enhances rhinovirus-induced toll-like receptor 3 expression and interleukin-8 secretion in A549 cells. Am J Rhinol Allergy 2009; 23: e5-e9.

29 Hoshino Y, Mio T, Nagai S, et al. Cytotoxic effects of cigarette smoke extract on an alveolar type II cell-derived cell line. Am J Physiol Lung Cell Mol Physiol 2001; 281: L509-L516.

30 Leist M, Jaattela M. Four deaths and a funeral: from caspases to alternative mechanisms. Nat Rev Mol Cell Biol 2001; 2: 589-598.

31 Carson JL, Lu TS, Brighton L, et al. Phenotypic and physiologic variability in nasal epithelium cultured from smokers and nonsmokers exposed to secondhand tobacco smoke. In Vitro Cell Dev Biol Anim 2010; 46: 606-612.

32 de Souza PM, Lindsay MA. Apoptosis as a therapeutic target for the treatment of lung disease. Curr Opin Pharmacol 2005; 5: $232-237$. 